\title{
EDITORIAL
}

\section{Pre-implantation genetic testing for hereditary pulmonary arterial hypertension: promise and caution}

\author{
Rizwan Hamid* and James Loyd ${ }^{\#}$
}

$\mathbf{P}$ ulmonary arterial hypertension (PAH) is a progressive, fatal disease and most PAH patients have a poor prognosis despite standard-of-care therapies. Heritable $\mathrm{PAH}(\mathrm{HPAH})$ is usually $(>80 \%)$ due to germline mutations in the bone morphogenetic protein receptor 2 (BMPR2) gene [14]. Thus, BMPR2 mutations constitute the largest known risk for developing PAH. One of the most perplexing features of $\mathrm{HPAH}$ is its reduced penetrance, which confounds genetic counselling. Nearly $80 \%$ of mutation carriers have no clinical symptoms, but they can produce offspring that are affected by HPAH [5]. Thus, disease development cannot be predicted in a mutation carrier and this deficiency in our knowledge about disease penetrance results in a significant physical, emotional and economic burden in HPAH families. Because $75 \%$ of subjects with HPAH have a New York Heart Association functional class rating of III or IV at the time of diagnosis, they have poor long-term prognosis: average life expectancy is $\sim 3$ yrs from the time of diagnosis [6], as the treatment options for $\mathrm{PAH}$, including $\mathrm{HPAH}$, continue to have no impact on the underlying pulmonary vascular disease and limited efficacy.

Thus, for individuals with mutation, the decision to have children is fraught with two major uncertainties. 1) Will the mutated gene be passed along to their child (a 50\% risk)? 2) If the mutation is passed along, will the child develop clinical disease? These important questions are not answerable with the current knowledge. The couple may decide to not have children, or can proceed to have children with the hope that their child will either not inherit the mutated gene or if they do, will hopefully never develop the disease. Pre-implantation genetic diagnosis (which has been used successfully in other genetic diseases), in theory, offers another welcome option that had not previously been considered in HPAH.

In this issue of the European Respiratory Journal, FrYDMAN et al. [7] show the successful use of pre-implantation genetic testing in a family with BMPR2-related HPAH. The BMPR2 mutation carrier was the father who, at the time of the procedure, did not have any clinical or echocardiographic evidence of PAH. In vitro fertilisation (IVF) was performed by intracytoplasmic sperm injection (ICSI) into a harvested ovum obtained after

*Dept of Pediatrics and Division of Genetics and Genomic Medicine, "Dept of Medicine and Division of Allergy, Pulmonary and Critical Care Medicine, Vanderbilt University School of Medicine, Nashville, TN, USA.

CORRESPONDENCE: J. Loyd, Dept of Medicine and Division of Allergy, Vanderbilt University Medical Center, T1219 vUMC North, 1161 21st Ave South, Nashville, TN 37232, USA. E-mail: Jim.loyd@vanderbilt.edu hormonal stimulation. Among seven embryos analysed, five carried the mutated BMPR2 gene and two did not. One of these unaffected embryos was implanted, leading to a successful pregnancy and the birth of a healthy child who did not carry the deleterious BMPR2 mutation.

The use of IVF is not unique, but this is the first time this procedure has been successfully used in HPAH. However, IVF in itself can be a less than ideal solution. In couples undergoing this procedure, there is a significant physical, emotional and financial (up to $\sim$ US $\$ 17,000$ per cycle) burden. Furthermore, IVF is not harmless and has been reported to be associated with complications including higher incidence of multiple births and a low but discrete risk of other genetic disorders. Several studies have shown that IVF performed by ICSI carries a higher risk of a major birth defect (in particular, genitourinary defects and epigenetic syndromes such as Beckwith-Wiedemann syndrome and Silver-Russell dwarfism) compared with a naturally conceived infant [6, 8-10]. The American Society of Reproductive Medicine considers ICSI a safe and effective procedure but notes that it may carry some increased risk for the transmission of selected genetic abnormalities to offspring [11].

On the surface, the case for using IVF in an autosomal dominant disease with poor prognosis and inadequate treatment options appears to be clear cut and would seem to offer no relative disadvantage for this procedure, yet many advantages. However, the use of IVF in this disease is complicated by many factors, including the fact that only $20 \%$ of mutation carriers will eventually develop disease. Furthermore, the use of ICSI for IVF introduces additional risk of unwanted and potentially severe genetic disorders. These two factors necessitate complicated and extensive genetic counselling provided by trained professionals along with careful genetic evaluation in early childhood. This may limit the number of couples who qualify or are willing to pursue this procedure. Regardless, the first successful application of this procedure in HPAH is an important forward step and offers a welcome additional option to a couple in which one partner carries a mutation.

\section{STATEMENT OF INTEREST}

None declared.

\section{REFFERENCES}

1 Cogan JD, Pauciulo MW, Batchman AP, et al. High frequency of BMPR2 exonic deletions/duplications in familial pulmonary arterial hypertension. Am J Respir Crit Care Med 2006; 174: 590-598. 
2 Cogan JD, Vnencak-Jones CL, Phillips JA 3rd, et al. Gross BMPR2 gene rearrangements constitute a new cause for primary pulmonary hypertension. Genet Med 2005; 7: 169-174.

3 International PPH Consortium, Lane KB, Machado RD, et al. Heterozygous germline mutations in $B M P R 2$, encoding a TGF- $\beta$ receptor, cause familial primary pulmonary hypertension. Nat Genet 2000; 26: 81-84.

4 Deng Z, Morse JH, Slager SL, et al. Familial primary pulmonary hypertension (gene PPH1) is caused by mutations in the bone morphogenetic protein receptor-II gene. Am J Hum Genet 2000; 67: 737-744.

5 Hamid R, Cogan JD, Hedges LK, et al. Penetrance of pulmonary arterial hypertension is modulated by the expression of normal BMPR2 allele. Hum Mutat 2009; 30: 649-654.

6 Chopra M, Amor DJ, Sutton L, et al. Russell-Silver syndrome due to paternal H19/IGF2 hypomethylation in a patient conceived using intracytoplasmic sperm injection. Reprod Biomed Online 2010; 20: 843-847.
7 Frydman N, Steffann J, Girerd B, et al. Pre-implantation genetic diagnosis in pulmonary arterial hypertension due to BMPR2 mutation. Eur Respir J 2012; 39: 1534-1535.

8 Hansen M, Kurinczuk JJ, Bower C, et al. The risk of major birth defects after intracytoplasmic sperm injection and in vitro fertilization. N Engl J Med 2002; 346: 725-7230.

9 Alukal JP, Lamb DJ. Intracytoplasmic sperm injection (ICSI) what are the risks? Urol Clin North Am 2008; 35: 277-288.

10 Alukal JP, Lipshultz LI. Safety of assisted reproduction, assessed by risk of abnormalities in children born after use of in vitro fertilization techniques. Nat Clin Pract Urol 2008; 5: 140150.

11 Practice Committee of American Society for Reproductive Medicine., Practice Committee of Society for Assisted Reproductive Technology. Genetic considerations related to intracytoplasmic sperm injection (ICSI). Fertil Steril 2008; 90: Suppl. 5, S182-S184. 